\title{
Correction to: A Multi-stage Representation of Cell Proliferation as a Markov Process
}

\section{Christian A. Yates ${ }^{1}$ (D) Matthew J. Ford ${ }^{3,4} \cdot$ Richard L. Mort $^{2}$}

Published online: 8 August 2019

(c) Society for Mathematical Biology 2019

\section{Correction to: Bull Math Biol (2017) 79:2905-2928 https://doi.org/10.1007/s11538-017-0356-4}

The original version of this article unfortunately contained a mistake. It has been corrected with this correction.

Equations (9) and (10) were transcribed incorrectly.

Equation (9) originally read

$$
\frac{\mathrm{d}^{k} M_{k}}{\mathrm{~d} t^{k}}=2\left(\frac{k}{C}\right)^{k} M_{k} .
$$

In fact, we should first have introduced scaled variables $m_{j}=M_{j} e^{k t / C}$, for $j=$ $1, \ldots, k$.

Equation (9) should then have read

$$
\frac{\mathrm{d}^{k} m_{k}}{\mathrm{~d} t^{k}}=2\left(\frac{k}{C}\right)^{k} m_{k} .
$$

The original article can be found online at https://doi.org/10.1007/s11538-017-0356-4.

$凶$ Christian A. Yates

c.yates@bath.ac.uk http://www.kityates.com

1 Department of Mathematical Sciences, Centre for Mathematical Biology, University of Bath, Claverton Down, Bath BA2 7AY, UK

2 Division of Biomedical and Life Sciences, Faculty of Health and Medicine, Furness Building, Lancaster University, Bailrigg, Lancaster LA1 4YG, UK

3 MRC Human Genetics Unit, MRC IGMM, Western General Hospital, University of Edinburgh, Edinburgh EH4 2XU, UK

4 Present Address: Department of Human Genetics, Rosalind and Morris Goodman Cancer Research Centre, McGill University, 1160 Pine Avenue West, Montreal, QC H3A 1A3, Canada 
Similarly, Eq. (10) originally read

$$
M_{j}=\left(\frac{C}{k}\right)^{k-j} \frac{\mathrm{d}^{k} M_{k}}{\mathrm{~d} t^{k}}, \text { for } j=1, \ldots, k-1 .
$$

It should have read

$$
m_{j}=\left(\frac{C}{k}\right)^{k-j} \frac{\mathrm{d}^{k-j} m_{k}}{\mathrm{~d} t^{k-j}}, \quad \text { for } \quad j=1, \ldots, k-1 .
$$

The equation should have been for the $m_{j} \mathrm{~s}$ and not the $M_{j} \mathrm{~s}$, and the order of the derivative was incorrect.

In addition, after Eq. (11), $z$ should be defined as "the first $k^{\text {th }}$ root of unity," not "the first $n^{\text {th }}$ root of unity."

The expression on the right-hand side of Eq. (16) is also missing a factor of $z^{r}$ in the numerator of the fraction inside the sum.

Originally, it read

$$
M(t)=\frac{1}{2 k} \sum_{r=0}^{k-1} \frac{2^{1 / k}}{2^{1 / k} z^{r}-1} \exp \left(\left(2^{1 / k} z^{r}-1\right) k t / C\right)
$$

However, it should have read

$$
M(t)=\frac{1}{2 k} \sum_{r=0}^{k-1} \frac{2^{1 / k} z^{r}}{2^{1 / k} z^{r}-1} \exp \left(\left(2^{1 / k} z^{r}-1\right) k t / C\right)
$$

In the top line of Eq. (25), the subscript $n$ on $\hat{M}_{n}$ should have been a $k$ leading to the corrected system

$$
\frac{d \hat{M}_{j}}{d t}= \begin{cases}\lambda_{1}\left(2 \hat{M}_{k}-\hat{M}_{1}-\hat{M}_{1} \hat{M}_{k}\right), & \text { for } j=1 \\ \lambda_{1}\left(\hat{M}_{j-1}-\hat{M}_{j}-\hat{M}_{j} \hat{M}_{k}\right), & \text { for } j \neq 1\end{cases}
$$

Since these were just transcription errors, they were corrected for in later equations and did not effect the results of the paper, but simply made it harder to follow the working.

Publisher's Note Springer Nature remains neutral with regard to jurisdictional claims in published maps and institutional affiliations. 